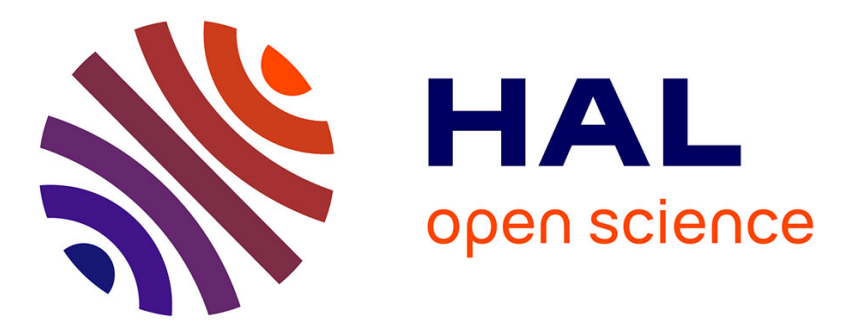

\title{
Le dialogue territorial au risque de l'écologie ? Traces et effets d'une concertation entre aménagements hydrauliques et restauration écologique
} Julie Riegel

\section{- To cite this version:}

Julie Riegel. Le dialogue territorial au risque de l'écologie ? Traces et effets d'une concertation entre aménagements hydrauliques et restauration écologique. Participations - Revue de sciences sociales sur la démocratie et la citoyenneté, 2018, 20 (1), pp.173-198. 10.3917/parti.020.0171 . halshs-03343516

\author{
HAL Id: halshs-03343516 \\ https://shs.hal.science/halshs-03343516
}

Submitted on 15 Sep 2021

HAL is a multi-disciplinary open access archive for the deposit and dissemination of scientific research documents, whether they are published or not. The documents may come from teaching and research institutions in France or abroad, or from public or private research centers.
L'archive ouverte pluridisciplinaire HAL, est destinée au dépôt et à la diffusion de documents scientifiques de niveau recherche, publiés ou non, émanant des établissements d'enseignement et de recherche français ou étrangers, des laboratoires publics ou privés. 


\section{Participations 2017/3 Le dialogue territorial au risque de l'écologie ?}

\section{Traces et effets d'une concertation entre aménagements hydrauliques}

et restauration écologique

Résumé : Pour contribuer au débat concernant les liens entre concertation et protection de l'environnement, cet article revisite un processus de dialogue territorial initié par un Syndicat de rivière pour la gestion d'un bassin versant soumis à de violentes crues. La mémoire des partiesprenantes et leur appropriation des accords passés révèle la qualité délibérative de ce processus, mais aussi le caractère à première vue minoré des ambitions écologiques de départ. La mise en œuvre opérationnelle des accords, de la mise en chantier à la réalisation des ouvrages, constitue en outre un révélateur autant qu'une mise à l'épreuve des acquis passés de la concertation.

Mots clés : concertation, eau, riverains, agriculteurs, délibération

\section{INTRODUCTION}

Depuis la décennie 1990, une corrélation implicite s'est établie entre démocratie participative et protection de l'environnement, à l'échelle nationale comme internationale. Ce postulat, que l'implication des citoyens à la décision publique favorise la prise en compte des enjeux environnementaux, se révèle notamment dans le principe 10 de la Convention de Rio, la Convention d'Aarhus, ou en France par la Commission nationale du débat public et la dernière Loi sur l'eau de 2006. Au regard des espoirs placés dans les dynamiques de concertation comme vecteurs de politiques environnementales ambitieuses, la persistance de la crise écologique, le retour d'une conflictualité antagoniste dans les projets d'aménagements, ou l'instrumentalisation des procédures de concertation, suscitent depuis quelques années des interrogations (Warin, Labranche, 2006), des désenchantements (Ballan, 2011; Dziedzicki, 2015) voire des remises en cause (Mermet, 2015). Cependant, un double tropisme sous-tend ces analyses : d'une part l'abondance de travaux centrés sur la temporalité des procédures de concertation (Fourniau et al., 2012), d'autre part la prééminence du Débat public comme focale d'analyse (Simard et al., 2006 ; Revel et al., 2007 ; Bourg, 2011 ; Archimbaud, 2016). L'exploration d'autres formes d'ingénierie de la concertation, plus confidentielles, moins institutionnelles et non réglementaires, peut contribuer à éclairer les liens entre la qualité démocratique d'un processus de concertation et sa valeur environnementale. C'est l'un des objectifs de la recherche anthropologique «Traces et mémoires des concertations territoriales environnementales $\gg .{ }^{1}$ Elle explore les perceptions des parties-prenantes quelques années après leur implication dans des processus de dialogue territorial, le devenir des accords qu'ils ont passés, ainsi que l'évolution des rapports de force ou de coopération entre acteurs concernés. La notion de "traces" d'un processus de concertation permet de s'extraire du registre de l'évaluation, en faisant l'hypothèse que tout dispositif de dialogue génère des résultats - espérés ou décevants -, mais aussi des effets non prévus qui demeurent invisibles et ignorés. Cela du fait de leur temporalité (ils se produisent après la fin du projet), ou parce qu'étant non planifiés car difficilement mesurables, ils échappent justement aux référentiels d'évaluation. Une trace, c'est «l'indice attestant qu'une empreinte s'est posée là, que quelque chose est réellement advenu » (Rancière, 2011, p. 476). Il y a par exemple trace d'une concertation environnementale si celle-ci est mise en récit par d'anciennes parties-prenantes, ou encore si les décisions prises se matérialisent par des aménagements dans le

1 Il s'agit d'une recherche postdoctorale de deux ans hébergée au Centre européen de sociologie et de science politique (CESSP/Université Panthéon-Sorbonne). Elle bénéficie du soutien de la Fondation de France et du partenariat avec l'association Geyser, spécialisée dans la formation et l'accompagnement au dialogue territorial. 
paysage ou induisent des changements de discours et de pratiques. Les archives de la concertation (comptes-rendus de réunion, rapports...), les documents institutionnels la contextualisant, ou encore des extraits de presse, sont également des traces matérielles. Des entretiens ethnographiques ouverts et des récits de vie constituent cependant la principale source de données de cette recherche. Ces méthodes d'interlocution permettent à l'acteur sollicité de cheminer librement dans différentes sphères - professionnelle, sociale, familiale... - et de s'inscrire dans différentes temporalités (Chaxel, Fiorelli, Moity-Maïzi, 2014). C'est ainsi l'acteur lui-même qui met en lumière les événements et les processus de changement qui font sens dans sa trajectoire personnelle et son contexte singulier. Cette option méthodologique est aussi un choix politique de l'anthropologue, qui postule que les acteurs ont des compétences et de la réflexivité, et peuvent devenir co-producteurs ou producteurs de savoir par la médiation du chercheur (Bensa, 2010). Dans cette posture de proximité, de confiance entre enquêteur et enquêté, il ne s'agit pas pour autant de coller au matériau biographique, qui peut proposer un «excès de sens », une mise en ordre trop parlante et trop linéaire, mais de repérer des «traits pertinents » (Passeron, 1990). Par son travail de compréhension, de croisement et de reconstruction de données qualitatives et polyphoniques, le chercheur choisit en effet de dialoguer avec certains éléments. Il s'approprie une toile de fond subjective dans laquelle il inscrit sa propre proposition intellectuelle, et choisit le statut qu'il donne à la parole recueillie -elle peut établir des faits ou les qualifier (Cerutti, 2011).

Parce que les paradigmes, les finalités, les méthodes des pratiques de concertation sont multiples et hétérogènes, cette recherche se focalise sur un type de concertation: le dialogue territorial environnemental. Celui-ci est mobilisé depuis les années 2000 par différents collectifs associatifs comme Geyser, l'IFREE, l'AFIP et les CPIE. Leurs animateurs collaborent de manière régulière, échangent des expériences, structurent et diffusent des savoirs et des savoir-faire, dessinant une communauté professionnelle de pratiques (Amin, Robert, 2008) ${ }^{2}$. L'approche du dialogue territorial contribue à construire une culture épistémique de la concertation environnementale (Knorr Cetina, 1999), qu'un collectif comme l'Institut de la concertation et de la participation capitalise pour influencer les politiques publiques. Processus de concertation itératif et s'étendant sur un pas de temps de plusieurs mois, le dialogue territorial embrasse pour ses promoteurs des phases de consultation, de concertation et de médiation, entendue comme une négociation assistée d'un tiers (Barret, Guihéneuf, 2017). La notion de concertation implique dans cette posture une intention coopérative de la part des parties-prenantes (Touzard, 2006 ; Beuret, 2012), ces dernières devant rassembler les catégories d'acteurs les plus concernées par l'objet de la concertation et représenter équitablement les groupes d'intérêts en jeu. Si on envisage la délibération comme un idéal de démocratie participative, où la légitimité d'une décision collective ne repose plus seulement sur qui la prend mais sur la façon dont elle est prise (Blondiaux, 2008), le dialogue territorial mobilise à trois égards des normes délibératives (Blondiaux, Sintomer, 2005 ; Bouvier, 2007). Il propose un cadre dialogique formel permettant aux groupes d'intérêts et aux citoyens concernés par un projet d'action publique de s'informer, de s'exprimer et de construire des propositions. De plus, les accords résultant de la délibération de toutes les parties prenantes ont une visée d'action explicite : il ne s'agit pas seulement d'éclairer un arbitrage public mais d'engager les parties-prenantes dans la mise en œuvre des accords passés. Enfin, les artisans du dialogue territorial encouragent des dispositifs déléguant un pouvoir de décision aux parties-prenantes de la concertation, à tout le moins que le processus décisionnel soit parfaitement transparent. En revanche, le dialogue territorial se démarque d'un format délibératif habermassien classique, qui implique de produire des arguments du registre rationnel et d'en débattre afin de confronter des opinions et de convaincre (Manin, 2002 ; Girard, Le Goff, 2010).

2 La plate-forme numérique COMEDIE http://www.comedie.org/ qui propose des ressources dédiées à la Concertation et la Médiation en Environnement, concourent à l'élargir et la rendre opérante. 
L'approche du dialogue territorial constitue ainsi un prisme stimulant et original pour interroger les liens entre aspirations démocratiques délibératives et démocratie écologique. Ces processus où la décision tend à être déléguée à un ensemble limité de parties-prenantes, éclairées par des expertises plurielles, invitées non à se convaincre mais à se comprendre, peuvent-ils contribuer à une démocratie écologique locale (Bourg, 2011) ? La focale du dialogue territorial sur la notion de contraintes et de besoins des acteurs les plus concernés par un projet ou une politique, permet-elle de construire de l'intérêt commun dans une approche suffisamment holiste (Bourg et Whiteside, 2010) ? Quatre expériences de dialogue territorial à revisiter ont été retenues pour cette recherche, relevant toutes de la gestion de l'eau et des zones humides, thème se prêtant particulièrement bien à l'analyse des effets des concertations territoriales environnementales. Le caractère décentralisé de la politique de l'eau en France depuis les années 2000 a en effet favorisé une approche subsidiaire généralement pilotée par des autorités non-étatiques (Le Bourhis, 2003). Elles sont censées négocier au niveau du territoire la mise en œuvre des prescriptions réglementaires nationales et des paradigmes de gestion des bassins versants promus par les agences de l'eau.

C'est le cas du Syndicat de rivières Brévenne et Turdine (SYRIBT), crée en 2006 par cinq communautés de communes recouvrant le bassin versant éponyme, dans les Monts du Lyonnais. Les lits et les berges des rivières Brévenne et Turdine appartiennent aux propriétaires riverains, mais les eaux sont considérées comme patrimoine de la nation. Ce bassin versant est marqué par un régime des eaux contrasté, qui combine de forts étiages estivaux, de hautes eaux hivernales, et des crues rapides et récurrentes, provoquant des inondations brutales et dévastatrices. Les agglomérations en aval du bassin versant, en particulier l'Arbresle (figure 1), ont été marquées par les crues de 1983 et de 2008, qui ont généré des dégâts matériels et psychologiques considérables ${ }^{3}$. En 2012, dans le cadre du second contrat de rivières, le Syndicat initie un projet ambitieux de "Restauration hydraulique et écologique du bassin versant Brévenne-Turdine » qui compte deux objectifs présumés conciliables : la protection contre le risque d'inondation par la construction d'ouvrages hydrauliques, et la restauration morphologique des rivières. Afin de favoriser l'acceptabilité des aménagements prévus, le Syndicat mandate la coopérative DialTer ${ }^{4}$, spécialisée dans le dialogue territorial, pour conduire une concertation, initialement envisagée comme une simple consultation ${ }^{5}$. Si la première partie de l'article analyse la qualité délibérative attribuée par les parties-prenantes au dispositif de dialogue, la seconde se penche sur le processus inattendu de minoration des objectifs écologiques initiaux, du moins tels qu'ils étaient planifiés par la rationalité initiale du projet. Le démarrage concret des travaux sur le bassin versant, conformément aux actions validées il y a maintenant plusieurs années, s'avère en outre être un révélateur autant qu'une mise à l'épreuve du processus historique de concertation, comme le démontre la dernière partie.

\footnotetext{
3Voir le documentaire en ligne «Ca n'arrive pas qu'aux autres », http://www.riviererhonealpes.org/news/l-inondation-del-arbresle-le-film

4La coopérative DialTer conçoit, anime ou supervise des processus de dialogue territorial. Ses médiateurs comptent parmi les fondateurs de l'association Geyser, partenaire de cette recherche.

5Je remercie vivement Philippe Barret, Betty Cachot, Pierre-Yves Guihéneuf et Jean-Luc Campagne pour leur contribution à cette enquête, ainsi que Loïc Blondiaux pour son éclairage critique quant à la première version de cette analyse.
} 


\section{CONTEXTE INITIAL ET MEMOIRE DU DEROULE DE LA CONCERTATION}

\section{Le syndicat de rivière, un initiateur de concertation légitime mais attendu}

Lorsque le Syndicat de rivières Brévenne et Turdine envisage fin 2012 de mettre en discussion son projet d'aménagements hydrauliques et de restauration écologique, il n'en a aucune obligation légale. Mais cette démarche lui semble précautionneuse au regard du contexte local et se présente comme peu risquée, car il dispose alors d'une bonne légitimité à conduire ce projet, qui répond à une demande politique et sociale croissante.

La création de ce Syndicat en 2006 bénéficie de l'arrivée de nouveaux élus en 2008, qui s'engagent dans la conception d'un second contrat de rivière pour le bassin versant. Alors que les politiques de l'eau des années 1980 sont axées sur la protection des inondations par des travaux de génie civil (construction de digues sur les berges, rectification et curage du lit des cours d'eau...), la décennie 1990 ouvre sur de nouveaux paradigmes hydrauliques et écologiques. L'heure est à la restauration physique et morphologique des cours d'eau, facteurs de continuité écologique piscicole et sédimentaire, mais aussi de gestion du risque d'inondation par la restauration de zones d'expansion libre des crues. La chargée de mission et le président du SYRIBT vont porter ces nouveaux paradigmes auprès des 45 maires du bassin versant et promouvoir une rhétorique de solidarité et de cohérence amont-aval. Le président du Syndicat alors en exercice est exploitant viticole, en production de type agro-environnemental, ancien maire, et précédent président de la communauté de communes de l'Arbresle, la plus concernée par les inondations et la plus dynamique économiquement. Bien inséré localement, ses qualités de conciliateur, ses convictions environnementales et sa modération sont reconnues. Mais le contrat de rivière prend une teneur particulière à la suite de la violente crue de 2008. L'association Tous Unis Contre Les Inondations (TUCLI) basée à l'Arbresle, en dormance depuis plusieurs années, reprend de la vigueur. Elle mobilise la presse, interpelle les élus, et porte plainte en 2009 contre le Syndicat, la Mairie de l'Arbresle et l'État. La problématique des inondations devient une priorité pour les élus du Syndicat. L'ambitieux projet de «Restauration hydraulique et écologique des rivières Brévenne et Turdine » est alors conçu avec un bureau d'ingénierie hydraulique, qui sous-traite la partie écologique à un autre bureau d'étude.

Ce projet phare se voulant d'utilité publique, permet au besoin d'exproprier des propriétaires riverains récalcitrants à la construction des ouvrages hydrauliques, et le Syndicat n'a pas d'obligation de conduire une concertation. Mais le lancement de nouveaux aménagements impactant du foncier agricole sur ce territoire est problématique : certains exploitants riverains de la rivière Turdine, potentiellement concernés par les ouvrages hydrauliques planifiés dans le projet, ont déjà été touchés par la création d'une zone d'activités et par le chantier de l'autoroute A89 reliant ici Lyon à Roanne. En missionnant la société coopérative DialTer pour animer un processus de concertation au premier semestre 2013, la chargée de mission du Syndicat souhaite surtout s'assurer d'une bonne communication sur le projet, et engager une consultation auprès des riverains pour favoriser son acceptabilité. Mais l'objet du dialogue va considérablement s'élargir, de même que le pouvoir de décision confié au principal organe de la concertation.

\section{Un projet initial bousculé par les parties-prenantes}

Le projet initial du Syndicat est bien balisé lorsque la concertation démarre. Cinq sites sur les rivières Brévenne et Turdine sont déjà identifiés, qui doivent accueillir quatre ouvrages de ralentissement dynamique et trois opérations de restauration écologique, selon des paramètres 
techno-scientifiques déjà définis ${ }^{6}$. Pour les médiateurs extérieurs en charge d'animer la concertation, les marges de manœuvre doivent d'emblée être revues afin de justifier la démarche. Cela implique de rééquilibrer la place de l'expertise technique dans la concertation, comme le révèle leur proposition de prestation: «l'étude [au sens de l'expertise externe] doit fournir aux instances de concertation des éléments de compréhension, puis des pistes de solution, mais doit laisser aux instances le temps de porter leur propre diagnostic, de solliciter des éclairages complémentaires, de proposer des alternatives, puis de choisir $\gg$ (DialTer, 2012).

L'examen des archives de la concertation montre une architecture complexe du dispositif, articulant trois niveaux de dialogue (figure 2) : un groupe principal de travail rassemblant les représentants des acteurs concernés par le projet; des réunions délocalisées associant les riverains sur les cinq sites pressentis pour le projet ; un comité de pilotage qui n'a finalement assuré qu'une validation des propositions du groupe de travail principal. Pour l'ingénieur hydraulique en assistance à la maitrise d'ouvrage, cette expérience s'est avérée inédite :

«L'enjeu principal au début, c'était d'identifier les éléments qui pouvaient bouger par rapport à la proposition initiale. Et c'est pas évident, souvent les bureaux d'études veulent faire avancer les solutions auxquelles ils croient. Pour moi c'était neuf, et pour mon bureau d'étude aussi - enfin, ce niveau de concertation je veux dire. Ensuite on a participé à pas mal de réunions, on nous demandait de faire des présentations techniques accessibles, et le mot qui revenait souvent était "le champ des possibles". Ça nous a demandé beaucoup de travail complémentaire pour élaborer à chaque fois différents scénarios. Heureusement, ça a été reconnu et financé par le Syndicat, sinon on n'aurait pas pu jouer le jeu » ${ }^{7}$.

Dès la première rencontre du groupe de travail, les parties-prenantes demandent que les bénéfices des ouvrages hydrauliques soient précisés au regard de leurs impacts écologiques et économiques. Pour la Chambre d'agriculture, l'enjeu est de minimiser l'emprise d'ensemble du projet sur le foncier agricole et de limiter les expropriations. L'expertise technique des bureaux d'étude est croisée avec celle des associations environnementales et des institutions publiques compétentes. L'analyse des coûts et des bénéfices inclue aussi les perceptions et les besoins remontant des réunions locales. Les bureaux d'études sont sollicités pour travailler des scénarios alternatifs soit à l'échelle du projet dans son ensemble, soit pour les sites en particulier. De nouveaux éléments sont ajoutés au cahier des charges : ne pas prélever les matériaux nécessaires aux chantiers sur les terres agricoles ; utiliser les enrochements de berges enlevés pour renaturer la rivière dans la construction des barrages... La Fédération de pêche se mobilise particulièrement pour préserver la continuité écologique piscicole et déterminer des mesures de compensation. Son représentant se pose en égal vis-à-vis du bureau d'étude et porte des propositions techniques pour diminuer l'impact écologique des barrages, au niveau de leur structure interne, de leur luminosité ou des matériaux utilisés.

Après six mois d'allers-retours entre groupe de travail principal, comité de pilotage, et réunions délocalisées, le projet a largement évolué. Trois sites seulement au lieu des cinq initiaux ont finalement été retenus. Ils doivent accueillir deux barrages écrêteurs de crue en place des quatre ouvrages modélisés initialement, ainsi que deux opérations de restauration écologique. L'emprise des nouveaux ouvrages sur la rivière et l'impact global sur le foncier agricole sont bien moindres.

Le dispositif de dialogue territorial piloté par DialTer s'est achevé en juillet 2013, mais un comité de suivi a été instauré, impliquant les maires des communes et des représentants des agriculteurs

6 Les ouvrages de ralentissement dynamique sont des barrages "écrêteurs de crue", qui n'entrent en activité que pour un certain niveau de montée des eaux, laissant passer l'eau en deçà. Les exploitants agricoles et les propriétaires riverains de ces aménagements sont déjà dans des zones inondables, puisqu'en bord de de rivière, mais ils seront en situation de surinondation potentielle s'ils sont situés en amont des ouvrages.

7 Entretien téléphonique, 16/09/2016. 
riverains concernés par le projet. Ce comité est chargé d'évaluer les besoins et les dégâts occasionnés en cas d'inondations dans la zone d'influence des barrages. Il va également préparer l'enquête publique liée au projet de l'automne 2015. En parallèle, le Syndicat a mis sur pied un réseau de sentinelles, c'est-à-dire de personnes réparties sur le bassin versant en charge de surveiller l'état des eaux, ce qui renforce l'appropriation collective de la gestion du risque d'inondation.

Paradoxalement, au regard de l'évolution du projet au cours de la concertation, d'anciennes partiesprenantes du groupe de travail principal et du comité de pilotage sont dubitatifs sur l'apport des médiateurs de la concertation à cette dynamique. Ils s'étonnent tout à la fois de leur discrétion dans le travail d'animation des réunions, et du caractère formel et répétitif du rappel des règles de dialogue. A l'inverse, si les riverains impliqués dans les réunions locales conservent une perception floue du dispositif d'ensemble de la concertation, ils ont en mémoire certains actes de médiation et font part d'un climat relationnel d'ensemble dans lesquels ils se sont sentis reconnus et respectés.

\section{«On nous a écoutés. On a pu discuter».}

Le représentant de la Chambre d'agriculture souligne en entretien le souhait affiché dès le départ par le Syndicat de rivières de ne pas passer en force auprès des riverains impactés par l'emprise des aménagements prévus. L'indemnisation juste des propriétaires et exploitants agricoles touchés par le projet est manifestement consensuelle pour les parties-prenantes. Le fait que ces acteurs vont en supporter les conséquences en amont, au bénéfice des agglomérations en aval, est reconnu. La rhétorique d'une nécessaire solidarité amont-aval au sein du bassin versant est d'autant plus délicate à manier qu'il est collectivement admis que l'importance des inondations dans les agglomérations est liée à des politiques d'urbanisation irresponsables, ayant multiplié les constructions en zones inondables. Le projet du Syndicat ampute ainsi pour la troisième fois le foncier de B.G. Éleveur laitier, il était jusqu'à peu autonome dans sa production de fourrage avec l'alternance de ses parcelles en prairies de pâture, de culture de maïs et de blé, sur 35 ha. Au moment de notre enquête, il vient de transmettre son exploitation à son fils, avec un foncier amputé de quatre hectares par le chantier d'autoroute, de 7 hectares par la nouvelle Zone d'Activités d'Actival, et d'environ $4000 \mathrm{~m}^{2}$ par le projet du Syndicat. Avec Actival, il a été assigné deux fois en justice au titre de la Déclaration d'Utilité Publique du projet, puisqu'il refusait les conditions proposées de rachat des terres et d"indemnisations de fermage. Il a encore en mémoire les trois hommes venus s'asseoir à sa table pour négocier : "On s'est sentis seuls ». Le vécu de cette expérience est douloureux, et livre en miroir un regard contrasté sur le dialogue avec le Syndicat :

«Il y a eu deux ans de discussions quand même avec le Syndicat. Il y a les conventions, sur les barèmes de la Chambre. Avec Actival, on a été traités comme des moins que rien. Avec l'autoroute un peu mieux : il y a eu des écrits cette fois (...). Ici, avec la rivière, bien sur ça n'a rien à voir. On a pu discuter. On s'appelle quand on veut. Et le temps. Ces choses là, il faut du temps. Vous trouvez pas ? Pour assimiler. Actival, on n'a pas été traités comme des personnes $»^{8}$.

Cet historique des projets d'aménagements sur le territoire a cependant généré de l'expérience, du savoir-faire. La Fédération de pêche du Rhône a testé des dispositifs techniques pour la franchissabilité piscicole sur le chantier d'autoroute. La Chambre d'agriculture du Rhône y a fait ses armes en matière de barèmes d'indemnisation, avec des négociations âpres avec Vinci. Le protocole qui a alors été passé sert de référence dans la région, et le Syndicat Brévenne Turdine a pu s'y référer. Le rachat des terres directement dans l'emprise des travaux a ainsi été validé à hauteur de

8 Entretien à St-Romain-de-Popey, 12/12/2016. 
2,50€ le $\mathrm{m}^{2}$, la même pour tous les riverains, contrastant avec la fourchette de 0,90 à $1,10 €$ des projets d'aménagement précédents. Le détail des indemnisations prévues est complexe, mais il faut en souligner d'une part la définition à l'amiable avec chacun des riverains, d'autre part l'effort de lisibilité et de traçabilité réalisé par le Syndicat. Il a rédigé une convention d'ensemble reprenant l'ensemble des accords négociés : la convention d'occupation temporaire, la convention de rachat, l'arrêté préfectoral de la servitude d'inondation... Il demeure à établir un acte notarial de ces accords, inscrit aux hypothèques, pour en valider le caractère juridique.

Au-delà des barèmes standards proposés par la Chambre, la situation singulière de chaque agriculteur riverain a été considérée : par exemple la prise en compte de la dégradation des clôtures par le passage d'engins, la perte de fertilité temporaire de parcelles occupées par les travaux de chantier, l'adaptation des indemnisations sur les servitudes d'inondation selon les assolements, le temps de décrue et de remise en état des parcelles, les circuits de déplacement des bêtes modifiés le cas échéant... M.D., dont deux hectares de terres sont actuellement occupés par le chantier de construction du premier barrage, a apprécié l'attitude du Syndicat dans ce projet. Il évoque également la présence de médiateurs à certains moment charnières :

«Le Syndicat nous a fait participer à tout le projet, de la pensée à la réalisation. On a visité un autre site, on a demandé notre avis sur tout, et c'était compliqué : des inondations aux accès, à la servitude... Ils ont demandé aux riverains. Il y avait des réunions générales pour tous les sites, et puis par site. Avec la Mairie. Au début, il y avait un médiateur, payé par le Syndicat mais qui notait ce qu'on demandait. C'était quelqu'un de neutre. Il demandait les conséquences pour nous. Parce que le Syndicat, il avait son projet quand même. Le médiateur il essayait de comprendre, il reposait la question. C'était quand même quelqu'un de simple, d'abordable, qui pouvait retenir vos questions, et revenir avec vers le Syndicat (...). Au début le Syndicat avait un peu peur avec les agriculteurs. Le médiateur c'était un tampon, c'est moins agressif de part et d'autre $»^{9}$.

Pour un autre type de riverain, le déroulé de la concertation et la mouture finale du projet résonnent aussi positivement. C'est le cas pour la présidente de l'association TUCLI, qui a été membre du groupe principal de travail. Sa maison est en bord de rivière, à l'Arbresle, elle fait partie des 260 familles de la commune sinistrées par la crue de 2008. Pour sa part, les assurances ont reconnu 110 $000 €$ sur $180000 €$ de dégâts estimés par une expertise externe à son domicile. Avec 40 adhérents de l'association, elle a porté plainte contre le Syndicat, la mairie et l'État en 2009. Les rapports de l'association TUCLI avec le Syndicat ont longtemps été houleux, la communication difficile. Articles dans la presse et interpellations incendiaires d'un côté, expulsion de ses représentants au sein de la commission du contrat de rivière de l'autre... Dans son récit transparaît sa colère passée, la perception des adhérents d'avoir été méprisés, perçus comme des riverains individualistes aux intérêts comptables. Malgré l'échec de leur action en justice, déboutée deux fois par le Tribunal administratif de Lyon, la présidente de TUCLI a le sentiment que ses intérêts et ceux des habitants en zone inondable ont enfin été pris en compte. La Mairie, le Syndicat, l'Agence de l'Eau, l'ONEMA, manifestent désormais de la considération à l'association. En outre, la mise en place sur le bassin versant d'un Plan de Prévention du Risque d'Inondation en 2012 et d'un Plan d'Action pour la Prévention des Inondations, sont lus comme une victoire. Jean-Pierre Le Bourhis (2007) souligne que le risque n'acquiert finalement une existence tangible, n'est "dit", que s'il est cartographié et hiérarchisé par les pouvoirs publics. Le discours de la présidente de TUCLI s'est également modifié au cours du dialogue, de la défense des intérêts particuliers des adhérents de son association, au

9 Entretien à St-Romain-de-Popey, 30/01/2017. 
registre de la gestion des eaux sur le bassin versant en général, puis à la prise en compte des impacts des ouvrages hydrauliques pour les agriculteurs. L'expression des motifs de mobilisation de TUCLI et des revendications de sa présidente ont évolué, gagnant en sens pour autrui et en légitimité (Barbier, Rémy, 2012). La mise en place de groupes de dialogue délocalisés sur les sites concernés par le projet, ce format délibératif «hors les murs », a visiblement favorisé l'expression et la compréhension de besoins ancrés dans des pratiques et dans un espace donnés. Dialoguer en des lieux situés a permis la prise en compte des situations spécifiques des agriculteurs riverains, qui ne se prêtent pas toujours à la verbalisation publique et à la mise en discours (Alphandéry, Fortier, 2005). Pour les riverains évoqués dans cette contribution, et à l'aune de leur micro-histoire dans ce bassin versant, ce processus de concertation a doté le projet du Syndicat de rivières d'une dimension de justice sociale, à la fois dans une composante économique redistributive, et dans une composante, plus prégnante, de reconnaissance (Fraser, 2011).

Au cours de la concertation, deux enjeux au départ difficiles à concilier ont été donc reconnus et appropriés par l'ensemble des parties-prenantes: la protection des agglomérations contre les inondations, et la préservation du foncier agricole avec une juste indemnisation des exploitants riverains. Les parties-prenantes ont constitué ce que l'équipe de médiateurs de DialTer appelle un intérêt commun, notion également mobilisée par la politologue Hélène Hatzfeld : "La référence à un intérêt commun ou collectif permet de désigner une attitude qui rassemble, fédère une diversité d'intérêts. (...) L'intérêt commun renvoie alors à la construction d'une orientation et non à l'existence de caractéristiques communes préexistantes » (20?). De plus, malgré l'asymétrie initiale entre le porteur du projet et les parties-prenantes en terme de pouvoir de décision, une marge de négociation réelle a finalement été conférée aux parties-prenantes. Selon la typologie de Laurent Mermet, cette concertation s'est déroulée selon un modèle «Proposer, Ecouter, Requalifier » (Mermet et al., 2004).

Mais au cours de ces étapes de requalification, les enjeux écologiques du projet semble être passés au second, puis au troisième plan. La restauration écologique des rivières Brévenne et Turdine, objectif initial aux côtés de la gestion du risque d'inondations, est moins visible dans les archives de la concertation, et peu audible lors des entretiens. Elle ne semble pas avoir acquis le statut d'intérêt commun.

\section{UNE MOTURE FINALE DU PROJET ÉCOLOGIQUEMENT MINORÉE}

\section{Hydrauliquelécologique : des résultats asymétriques}

L'examen des sites et des actions retenus au terme de la concertation rendent perplexes au regard des ambitions de départ en matière de restauration écologique. Lors de la premier réunion du groupe principal de concertation, des attentes sont pourtant patentes : "Une approche différenciée de la restauration écologique selon les bassins versants est nécessaire »; «Libérer les contraintes de la rivière sur la Turdine »; «Redonner de la place au cours d'eau sur la Brévenne »; « Fixer le niveau d'ambition à atteindre en matière de restauration écologique $\gg^{10} \ldots$ Mais les opérations de restauration écologique finalement validées à l'issue du dialogue portent sur deux sites. Le premier concerne la restauration d'un kilomètre de linéaire sur la Turdine, avec la rénovation d'un passage à gué, l'aménagement d'un seuil ${ }^{11}$ pour le passage des poissons, la suppression d'enrochements sur les berges et leur reprofilage en pente douce avec revégétalisation. Cette restauration doit permettre de retrouver une continuité écologique sur cette portion de rivière et restaurer une morphologie plus naturelle de son lit. Le second site concerne l'aménagement sur la Brévenne d'un seuil sous-tendant

10 Compte-rendu issu de la rencontre de février 2013.

11 Un seuil est un ouvrage de bois, de sable ou de pierres construit transversalement dans le lit d'un cours d'eau. 
un passage routier, qui entrave la remontée des poissons en période de frai. A l'aune des objectifs initiaux du projet, ce volet de restauration écologique apparaît dérisoire, surtout en comparaison du volet hydraulique.

Initialement, une opération devait à elle seule assurer l'équilibre d'ensemble du projet entre ses volets hydraulique et écologique : un chantier ambitieux sur la Brévenne pour renaturer plus d'un kilomètre de cours d'eau d'un seul tenant, sur une portion de la rivière très abîmée. Mais la concertation locale s'est mal passée sur ce site, marquant l'ensemble des parties-prenantes présentes. L'un des agriculteurs riverains a violemment manifesté son rejet du projet lors de sa présentation formelle en mairie, puis a perdu tout contrôle lors du déplacement des participants sur le terrain. La confrontation avec l'émotion violente de cette personne ponctue les entretiens réalisés auprès des parties-prenantes. Dans un contexte de passation de l'exploitation entre père et fils, où l'enjeu de transmission d'un capital foncier intègre était pour lui fondamental, le bureau d'étude a mis en avant un scénario spécifique impliquant l'empiétement des parcelles les plus rentables de l'exploitant, celles qui étaient plates dans cette vallée si encaissée. Une médiation de conflit avec un intervenant dédié de DialTer a ensuite permis de restaurer une discussion et d'entrevoir jusqu'à six scénarios scénarios alternatifs. Mais d'autres contingences sont apparues. Le scénario le plus consensuel s'avérait finalement assez faible au regard de ses bénéfices écologiques, pour un coût multiplié par quatre. D'un commun accord le groupe principal de travail a décidé de suspendre temporairement la restauration écologique de ce site. Puis le fils de l'agriculteur riverain concerné s'est alors tué dans un accident de voiture.

Au-delà de la situation tragique de cet exploitant riverain, le dispositif de concertation semble néanmoins avoir été moins "resserré" sur le site en question. La problématique des inondations, soumise à une pression politique et sociale forte, a fortement pesé sur l'équipe du Syndicat, sans compter la complexité des dimensions administratives, réglementaires et financières liées à l'élaboration des ouvrages hydrauliques. Une asymétrie dans la délibération sur les objectifs et les choix techniques semble s'être opérée entre le volet hydraulique et écologique du projet. Sur les cinq concertations délocalisées, ce site était en outre le seul à n'embrasser qu'une dimension écologique et non hydraulique. Dès lors la place du bureau d'étude écologique était exclusive. Il pouvait enfin déployer une ingénierie écologique ambitieuse et complexe. Son représentant a pris une posture prescriptive et en surplomb, ce que les porteurs de la concertation avait jusque là évité de la part des experts extérieurs.

Cependant, la perte d'ambition finale du projet au regard de ses ambitions écologiques initiales tient aussi au retrait, des représentants des associations environnementales. La renaturation des berges des rivières et la restauration morphologique de leurs lits ont dans l'ensemble eu peu de porte-parole (Latour, 1999).

\section{La restauration écologique, un manque de porte-parole?}

Lors de notre enquête, certaines parties-prenantes s'avèrent dubitatives au regard du double objectif initial du projet du Syndicat - la construction d'ouvrages hydrauliques et la restauration écologique. Pour certains pêcheurs, ce projet est incohérent: il s'agit en fin de compte d'enlever des enrochements de berges pour les remettre plus haut dans des ouvrages. Les représentants des organisations environnementales ont également perçu ce projet comme principalement dédié à la protection contre les inondations, imposé par la demande sociale. La dimension écologique leur semble d'emblée biaisée, tant les barrages n'ont aucune vocation environnementale, et bien que ce biais leur paraisse légitime. Les deux barrages vont artificialiser des portions de la Turdine en bon état écologique, et nécessairement modifier la dynamique liquide et solide de la rivière. Et puis les propositions techniques en matière écologique laissent circonspects certains acteurs : les seuils 
constituent-ils vraiment une entrave à la circulation piscicole, sur des rivières qui justement entrent en crue ? Quant à la renaturation des berges de la Turdine, avec l'enlèvement des roches apposées il y a plusieurs décennies pour diminuer l'érosion, la replantation de végétaux va t-elle suffire à les stabiliser?

Pour autant, ni les riverains, ni les représentants associatifs, n'ont transformé leur réserve en opposition au projet. L'un des médiateurs de DialTer évoque la posture des associations lors de la concertation : "C'est comme s'il y avait d'entrée de jeu une sorte de consensus, que ce projet était pour la sécurité des personnes et des biens. Elles ne se sont pas positionnées sur d'autres alternatives $»^{12}$. C'est sur les mesures de compensation liées aux barrages que ces acteurs se concentrent, afin qu'elles soient à la hauteur des dommages. Ce peu de militantisme écologique, cette posture conciliante sur le projet, sont liés aux relations anciennes et amicales de ces acteurs avec le Syndicat, qui est globalement perçu comme actif en matière écologique. De plus, pour les organisations environnementales, l'enjeu sur le bassin versant est ailleurs : il concerne la gestion quantitative de l'eau et les problèmes d'étiage, certains cours d'eau étant complètement à sec en saison estivale. En toile de fond se profile une controverse récurrente avec la profession agricole, et notamment avec la Chambre d'agriculture, sur la question des retenues collinaires ${ }^{13}$ qui jalonnent le bassin versant et ne respectent pas l'obligation de débit réservé. Sur d'autres scènes de concertation convoquant cet enjeu, la conflictualité est forte et les représentants associatifs sont pris à parti parfois violemment. Le peu de militantisme de certaines parties-prenantes semble lié au soulagement de vivre une concertation sans animosité, comme le traduit ce représentant associatif :

« Finalement, sur ce projet, au-delà des résultats sur lesquels j'ai peu d'attentes, c'est la concertation elle-même le plus positif. Il y a la fausse concertation ; et la concertation bienveillante qui s'est faite là. On a vu en réunion que les processus de communication non violentes sont bien plus efficaces que de pseudo-concertations. Je n'ai pas réalisé tout de suite, je ne me suis pas vraiment rendu compte, que ces principes étaient là //. Si on est considéré, et si nos enjeux sont considérés, on a moins tendance à vouloir porter son truc à tout prix $»^{14}$.

Dans ce projet, la restauration écologique est sous-tendue par un discours technico-rationnel généralisant, rattaché à des prescriptions et des normes publiques environnementales surtout véhiculées par l'agence de l'eau. Ces prescriptions ne convergent pas avec un discours d'attachement sur le registre du proche et du sensible, ou encore de l'esthétique, de l'héritage, toutes motivations possibles d'un discours de sensibilité écologique (Bozonnet, 2012). L'historique de ce bassin versant éclaire cette absence de patrimonialisation des cours d'eau. La rivière Turdine a longtemps été considérée comme une rivière industrielle : le développement des teintureries en amont du bassin dans la décennie 1980 a engendré une pollution croissante due aux rejets de nitrates et de phosphore. Quant à la Brévenne, elle n'a cessé d'être endiguée et corsetée entre la route nationale, la voie ferrée et l'urbanisation. Ce peu de porte-parole du volet écologique est aussi le fait des élus et des institutions publiques impliqués dans la concertation, confrontés au fait que la restauration écologique des berges et des lits des rivières manquent manifestement de légitimité sociale. Ces acteurs préfèrent la stratégie du cheval de Troie, couplant des actions de restauration écologique avec des opérations hydrauliques, dans une rhétorique d'ensemble de gestion du risque. Certains élus du Syndicat craignent en outre d'être perçus comme une structure trop technocratique, vectrice

12 Entretien téléphonique, 15/12/2016.

13 Ces retenues artificielles, principalement alimentées par les eaux pluviales et les ruisseaux, sont utilisées essentiellement pour l'irrigation des cultures ou l'alimentation des canons à neige en montagne.

14 Entretien à Lyon, 10/10/2016. 
de prescriptions administratives et réglementaires verticales. De plus, d'un point de vue pratique, les leviers juridiques ne sont pas les mêmes pour un projet de gestion du risque d'inondation ou de restauration écologique. Cette dernière pouvant difficilement être reconnue d'utilité publique, elle impose aux maîtres d'œuvre des négociations incertaines avec les riverains.

En outre, la difficulté de qualifier un projet d'utilité publique au regard du droit positif biaise la mise en œuvre des politiques environnementales. Dans son analyse de l'application de la Loi Littoral et de la Loi Montagne, Pierre Lascoumes (1995) montre le rôle modeste de la règle de droit comme modérateur des conduites, «le système légal agissant principalement comme une fiction opératoire ». La protection de l'environnement n'est pas posée comme une valeur supérieure des politiques publiques environnementales, déclinée par des objectifs concrets, mais comme le résultat de conciliations et d'ajustements entre différents intérêts, discutés selon des procédures ou des processus souvent dévolus à des instances locales.

Le fait que les parties-prenantes n'aient pas adhéré à l'ensemble des options proposées, et retenues, ne décrédibilise pas pour autant ce processus de concertation, si on considère avec Bernard Manin que la légitimité de la décision ne réside pas dans l'expression de toutes les volontés individuelles car l'unanimité est illusoire, mais dans la délibération de tous (1985). D'ailleurs à l'automne 2015, les accords issus du dialogue territorial ont été largement publicisés et mis à l'épreuve par l'enquête publique relative au projet. Celle-ci a reçu le soutien des associations environnementales partiesprenantes de la concertation, et n'a pas soulevé d'opposition de la part des riverains du projet. Un « intérêt général territorialisé » a bien émergé du processus de dialogue, impliquant une hiérarchisation progressive des enjeux, au cours de laquelle la perte d'ambition en matière de restauration écologique a été balancée par des mesures de compensation plus poussées sur le volet hydraulique (Beuret, Cadoret, Rey Valette, 2016).

Le démarrage concret à l'automne 2016 des travaux de construction des ouvrages hydrauliques et des opérations de restauration écologique, concomitant à notre enquête, constitue dans ce contexte un révélateur autant qu'une mise à l'épreuve des accords et des décisions issus de la concertation. Cette mise en œuvre est source d'imprévus, d'incertitudes et de retour de la conflictualité, éclairant d'un jour nouveau les acquis passés de la concertation.

\section{LA CONCRETISATION DES DÉCISIONS, UNE ETAPE PERILLEUSE}

\section{De nouveaux chantiers sur la rivière : dépossession et incertitude}

Les trois exploitants agricoles directement touchés par les travaux sur la Turdine ont tous hérité de la ferme de leurs grands-pères, qui avaient loué ou acquis ces terres abritant jadis des moulins, au Marquis d'Albon, dont le château est encore visible en surplomb de la rivière à Saint-Romain-dePopey. Le Marquis avait attribué les exploitations selon un principe d'équité : 35 à 40 hectares par exploitant, avec pour tous certaines parcelles en bord de rivière, considérées comme les plus rentables. Les inondations représentent des aléas familiers pour les agriculteurs riverains de la Turdine et un risque ancien, accepté et approprié, corollaire pour disposer de parcelles fertiles, limoneuses, et d'un accès à l'irrigation privilégié. Même si la Turdine semble monter «plus vite, plus fréquemment et plus fort » depuis dix ans, les conséquences de ses débordements sont connus et variables pour ces exploitants : ravinage des berges, inondations des bâtiments de ferme, dépôt de matériaux à enlever... Les familles ont toujours fait face à ces aléas naturels avec un certain isolement, et des coûts humains et matériels non négligeables. Les indemnisations publiques sont aléatoires et dépendent du statut de catastrophe naturelle attribué ou non à l'événement par la Préfecture. La mise en place de servitudes d'inondation avec le Syndicat offre dès lors une garantie d'indemnisation nouvelle pour ces riverains, ainsi qu'un accompagnement, une prise en charge 
collective dès qu'un risque de crue se présente. Pourtant, les nouveaux aménagements sont une source d'inquiétude et de tensions nouvelles avec le Syndicat. Elles révèlent en creux les liens de ces riverains avec la rivière.

« On va perdre nos repères » répète plusieurs fois B. G. en entretien. Les épisodes de crue, il les a aussi vécus, mais ses prairies sont faiblement inondées et peu de temps, grâce à ses pratiques culturales qu'il met en avant: peu de labour, une fumure auto-produite, la présence de vie microbienne et de vers de terre qui façonnent un sol aéré et perméable. Celui-ci absorbe l'eau, s'expose peu au lessivage et se régénère vite après les inondations. Ce qui l'inquiète dans la construction des barrages, c'est de ne plus comprendre la rivière, le processus de crue, de ne plus pouvoir l'anticiper et agir en fonction de manière autonome. Ainsi, jusqu'à présent, il s'appuie sur des repères auditifs : ce bruit de la rivière qui gronde, qui monte, que la présidente de l'association TUCLI, bien plus bas, évoque elle aussi. Il sait aussi que la fuite des rats qui quittent les berges est à prendre en compte. Ces éléments parmi d'autres, lui permettent de s'organiser pour déplacer ses bêtes et prévoir le fourrage nécessaire. Mais le barrage va bouleverser tout cela : en combien de temps l'eau montera, et de quelle façon sur ses prés ? De plus, si les indemnités lui permettent de racheter d'autres parcelles et même de payer les frais notariaux, son exploitation perd globalement en rentabilité, et lui-même en autonomie comme en qualité de vie. Le foncier agricole disponible dans sa commune est de plus en plus rare, à louer comme à racheter, tandis que son exploitation historique d'un seul tenant générait des économies d'échelles en temps de travail et en coûts de déplacement, et facilitait les innovations culturales.

Le démarrage des chantiers, c'est aussi l'irruption chez soi des machines, des ouvriers de chantier, et d'un nouvel interlocuteur, l'entreprise Vinci. Les riverains ont peu de prise sur ces intrus. Ainsi cet exploitant aurait voulu voir précisément les plans détaillés de la restauration écologique sur ses berges : combien de mètres cubes de terre et d'enrochements, et où, allaient être prélevés ? Et quels végétaux replantés? Une certaine méfiance envers les experts est ici palpable : cet acteur a par exemple fait vérifier les cotes topographiques prises par le bureau d'étude sur son terrain. Il répète à plusieurs reprises : «c'est quand même chez moi », «ils sont chez moi ». Quant à son voisin, il s'est alarmé de l'arrivée de Vinci sur l'hectare de terre qu'il met à disposition temporairement pour le chantier : le décaissement et la mise en sécurité de la couche supérieure de terre arable, avant occupation des lieux, n'avait pas fait l'objet d'un accord signé avec le Syndicat. Le jour prévu de l'arrivée des ouvriers, il barre l'accès de son champ avec son tracteur. L'affaire se règle à l'amiable le lendemain.

Les travaux, ce sont aussi des imprévus qui ont échappé aux modélisations techniques, aux dires des experts, aux paramètres mis en dialogue durant la concertation. Ainsi le 22 novembre 2016, alors que les travaux du premier barrage ont plusieurs semaines de retard et que l'opération de restauration écologique de la Turdine vient de s'achever, la pluie s'abat pendent douze heures. La montée des eaux atteint le niveau d'alerte entre les communes du bassin versant. Le jour suivant, il y a peu de dégâts dans les agglomérations en aval, mais des impacts sur les zones des chantiers. La crue a notamment touché l'opération de restauration écologique juste achevée. En rive gauche, les berges venaient d'être libérées de leurs enrochement, pour redonner au cours d'eau un espace de libre expansion. La rivière s'y est engouffrée avec violence, emportant $400 \mathrm{~m} 3$ de terre, qu'elle aurait $\mathrm{du}$ éroder progressivement sur dix ans d'après les modèles du bureau d'étude écologique. L'exploitant riverain et son fils sont terriblement en colère : désormais la nouvelle berge est au bord des parcelles de maïs. En cette période où le sentiment de déposession était déjà vif, il est hors de question pour ces riverains de laisser la situation en l'état. Ils exigent que les enrochements antérieurs soient remis en place.

Avec la mise en chantier du projet, les riverains passent d'une situation de risque à une situation d'incertitude (Callon, Lascoumes, Le Galès, 2001). La technique est génératrice d'incertitude, notion 
bien distincte de celle du risque. Le risque relève d'un danger identifié, associé à l'occurrence d'un événement ou d'une série d'événements, parfaitement descriptibles. Alors que l'incertitude ôte la possibilité d'anticipation, de poser des choix, au regard de l'ignorance de l'état du monde à venir.

\section{Une dynamique relationnelle fragilisée}

Dans le cas des dégâts évoqués sur le site de restauration écologique, c'est l'acteur le plus extérieur au territoire qui concentre dans un premier temps la colère des riverains : le bureau d'étude écologique, et à travers lui les savoirs experts :

«Ils ont fait venir des gens pas d'ici, ils viennent peut-être du Nord, de Lille, de Paris. Ils auraient mieux fait de demander aux anciens, ça aurait coûté moins cher ! Ils ont jamais vu la rivière en crue si ça se trouve, la force. (...) On leur demande de stabiliser une berge, et y a rien qui tient ! Et après ils doivent faire une digue [un barrage], ça fait peur. (...) Ils ont la théorie, ce sont des modèles. Mais y a pas une rivière qui ressemble à une autre ${ }^{15}$.

Le temps passant, cette colère se déplace petit à petit sur le Syndicat ; la situation se crispe d'autant plus que l'élaboration d'une réponse prend du temps, nécessitant devis, demandes d'autorisations administratives, interfaces avec le bureau d'étude écologique. Pendant plusieurs semaines, la communication est pratiquement rompue, les représentants du Syndicat sont invectivés, l'ensemble des accords passés remis en cause. Pourtant, cet exploitant riverain a largement participé au groupe principal de travail de la concertation, mais sous un autre statut : il était à l'époque partie-prenante en tant que maire, et en tant qu'élu du Syndicat. Même s'il dispose d'une vision d'ensemble du processus de dialogue, de ses étapes, et des choix posés sur le projet, la position et les besoins de cet acteur ont considérablement évolué, et c'est d'abord en tant que riverain qu'il se manifeste ici. Son «identité d'action» est plurielle (Lascoumes, Le Bourhis, 1998): dans un processus de concertation, un acteur est protéiforme, il revêt différentes identités qui se superposent et ne s'expriment pas de la même façon si le contexte et les systèmes d'alliance dans lesquels il agit se transforment. En outre, face à l'ensemble des imprévus décrits ci-dessus, et aux travaux qui envahissent leur propriété privée et leur outil de travail, les riverains concernés sont plutôt esseulés : la dimension collective inhérente à la période de la concertation s'est achevée, le portage commun du projet s'est étiolé, d'autant plus que la restauration écologique n'a pas acquis la dimension d'un intérêt commun.

Le manque de prise des riverains sur le déroulement des chantiers d'aménagements, et leur perte de visibilité sur la gestion des inondations, semblent s'ajouter à un sentiment global de perte d'autonomie. En tant que propriétaires riverains, ils disposent de droits individuels historiques, mais ces droits sont de plus en plus encadrés et contrôlés par l'administration. L'entretien des berges, la taille et le prélèvement des boisements, le prélèvement d'eau, nécessitent des déclarations régulières et des autorisations auprès de différents corps administratifs. M. D. a ainsi préféré creuser un étang sur sa propriété pour abreuver ses vaches afin de s'affranchir de la tutelle administrative, ce qui a déclenché une visite impromptue de la police de l'eau. L'administration dispose d'un pouvoir réglementaire et envisage les eaux courantes comme un bien commun (res communis), dont la préservation est d'intérêt général. Alice Ingold (2011) a montré les liens et les tensions depuis le 19e siècle entre les droits juridique et administratif, et les conflits de savoirs sur la gestion des eaux qu'ils véhiculent, relevant soit d'une histoire socio-écologique et territorialisée, soit d'une approche technique et scientifique des cours d'eau.

Le surgissement de la conflictualité et la récurrence des imprévus entraînent certaines parties-

15 Entretien informel, Bully, 13/12/2016. 
prenantes à douter des options validées lors de la concertation, ou encore de la qualité de la délibération sur le volet de restauration écologique. L'un des salariés du Syndicat se questionne :

«On n'a pas assez travaillé une vision commune de la rivière, et ce qu'impliquait la restauration écologique. On a pêché aussi par fatigue, en se concentrant sur les questions liées aux barrages, le besoin d'information. En se disant que finalement la restauration écologique sur ce site était acquise, qu'elle ne posait pas de problème, qu'elle était accessoire. Alors que non, pas du tout $»^{16}$.

Pourtant, cette période donne également à voir d'autres traces du processus de concertation. Plusieurs parties-prenantes soulignent la qualité des relations avec les représentants du Syndicat, et défendent la réputation de ses techniciens. La présidente de TUCLI capitalise sa compétence civique en compétence politique (Talpin, 2010) : entrée dans la liste d'opposition de la mairie de l'Arbresle, elle s'est battue pour être au moins suppléante au comité syndical, et met abondamment en récit sa nouvelle appréhension des dossiers du Syndicat, et son estime pour le travail des salariés depuis plusieurs années. Les réactions de ces derniers révèlent en outre leur acquisition de compétences de médiation, en terme de posture et de méthodes de dialogue. Ils manifestent en premier lieu de l'empathie et de la compréhension vis-à-vis des riverains concernés par les travaux en cours, et une capacité à encaisser la conflictualité et les débordements émotionnels. Dans un second temps, des outils de rétablissement du dialogue et d'une dynamique de négociation sont mobilisés. Certains salariés du Syndicat dotent ainsi leur structure intercommunale non plus d'une légitimité de représentation, mais de proximité et d'impartialité, au sens où Pierre Rosanvallon les mobilisent (2008). L'impartialité est une qualité, un comportement, attribué à des personnes ou des actions, qui renvoie au désintéressement, avec une attention portée à tous. La responsable du Syndicat, étroitement associée à la conduite du dispositif de concertation, note aussi l'influence de cette expérience de concertation dans ses pratiques de travail :

«Un des apports de DialTer c'est la prise de recul. Un opposant à un projet a ses raisons, ce n'est pas nous contre eux. Avant je prenais les choses comme un échec, personnellement. Mais si le projet ne se fait pas, c'est pas la mort. Dans ce genre de démarche, il y a un attachement qui se fait au processus, aux gens, bientôt plus qu'au projet lui-même $»^{17}$.

\section{Conclusion}

Les décisions prises à l'issue de ce processus de dialogue territorial relèvent manifestement d'un mécanisme de consensus apparent, décrit par Philippe Urfalino (2007). Il se produit lorsqu'une proposition ne génère plus l'expression d'un désaccord, ce qui ne signifie pas une unanimité fermement établie, mais le choix de certaines parties-prenantes de ne plus manifester de dissensus à un moment donné, pour des raisons variables. Il est manifeste que dans ce dialogue, le volet écologique du projet a souffert d'une asymétrie de légitimité initiale au regard des enjeux hydrauliques. Mais les acteurs environnementaux ne se sont pas efforcés de produire des arguments pour résorber cette iniquité de départ, peut-être du fait que la nature des intérêts des non-humains est difficile à définir, à mettre en partage, et revêt alors la forme d'un impératif moral se prêtant mal à la négociation et à des ajustements progressifs (Bourg, Whiteside, op cit).

En première lecture, cette concertation n'a donc pas permis de construire un intérêt commun relevant du volet écologique, mais un tel constat est réducteur, car biaisé par un point de vue 
naturaliste et hydrologique. Le caractère initialement binaire du projet, structuré en un volet hydraulique censé être purement anthropocentré, et un volet écologique purement éco-centré, reflète surtout les thématiques fléchées par les bailleurs de fond. Or le projet dans sa mouture finale s'avère bien moins consommateur de foncier agricole et de prairies de pâture que dans sa version initiale, et la continuité écologique dans les ouvrages hydrauliques y est mieux prise en compte. Le cadre initial de ce dialogue, corseté par la commande de départ, n'a pas donné aux parties-prenantes la marge de manœuvre pour questionner l'énonciation des enjeux écologiques du projet ni le périmètre territorial à considérer. Les parties-prenantes ont été sollicitées pour délibérer sur les solutions proposées par le projet et pour en négocier ses externalités.

Par ailleurs, le «point de passage transactionnel» d'une concertation, moment de bascule vers la conflictualité ou la coopération, décrit par Jean-Eudes Beuret dans des cas de négociations en bilatéral, se révèle instable dans notre cas d'étude. Il montre en revanche que l'expérience du dialogue territorial a modifié la perception de cette conflictualité par les porteurs de projets, et leur capacité à y faire face. En acceptant le surgissement de nouvelles tensions, la remise en cause des accords passés, et des phases imprévues de négociation, le Syndicat augure d'une capacité à passer d'une conflictualité antagoniste à une conflictualité agonistique, pour reprendre les termes de la philosophe Chantal Mouffe (2016). Les acteurs en conflit ne sont plus perçus comme des ennemis, mais comme des adversaires temporaires, porteurs de valeurs, de visions du monde, d'intérêts différents mais reconnus, avec lesquels il s'agit de dialoguer de manière démocratique et non autoritaire.

Title : A local deliberation ecologically risky?

Summary : This paper contributes to the discussion regarding the links between deliberation and protection of environment with a focus on a dialogue initiated by a local public actor for the management of rivers. We analyse the stakeholders's perception and apppropriation of the agreements achieved several years ago. Paradoxically, the quality of the deliberation have results in an undermining of the initial ecological goals, poorely argued by the environmental representatives. Moreover, the present works result from the agreements challenge the achievements of the deliberation process.

Key-words : deliberation, mediation, water management, farmers, riverside resident

\section{Bibliographie :}

Alphandéry P., Fortier A., 2005, «Les savoirs locaux dans les dispositifs de gestion de la nature » in L. Bérard, M. Cegarra, M. Djama, S. Louafi, P. Marchenay, B. Roussel, F. Verdeaux (dir.), Biodiversité et savoirs naturalistes locaux en France, Paris, INRA, p. 158-165.

Amin A., Roberts J., 2008, «Knowing in Action: Beyond Communities of Practice », Research Policy 37, p. 353-369.

Archimbaud J., 2016, Introduction au Carrefour-Débat « Débat public, concertation, conciliation, médiation : quelles synergies ? », 8 noembre 2016, CNDP, Paris.

Ballan E., 2011, « Démocratie et environnement, un mariage arrangé ? », Territoires, mars 2011.

Barbier R., Rémy E., 2012, «Les controverses environnementales publiques », in N. Lewis, R. Barbier, P. Boudes, J.P. Bozonnet, J. Candau Bozonnet, M. Dobré, F. Rudolf (dir.), Manuel de Sociologie de l'environnement, Presses de l'Université de Laval, p. 187-202.

Barret P., Guihéneuf P.Y., 2012, Guide pratique du dialogue territorial : concertation et médiation 
pour l'environnement et le développement local, La Tour d'Aigues, éditions de l'Aube.

Barret P., Guihéneuf P. Y., 2017, « A Territorial Dialogue in France », in C. Choquette, V. Fraser, (dir.), Environmental Mediation Across the World, Routledge, Québec, à paraître.

Bensa A., 2010, Après Levi-Strauss, pour une anthropologie à taille humaine, Textuel.

Beuret J.E., 2010, «De la négociation conflictuelle à la négociation concertative : un "Point de Passage Transactionnel" », Négociations 2010(1), n 13, p. 43-60.

Beuret J.E., 2012, « Mieux définir la concertation : du pourquoi au comment », Négociations 2012(1), n 17, p. 81-86.

Beuret J.E., Cadoret A., Rey Valette H., 2016, « Développement durable en zones côtières : comment territorialiser l'intérêt général environnemental ? Un cadre d'analyse », Développement durable et territoires [En ligne], 7(3), Décembre 2016.

Blondiaux L., 2008, Le nouvel esprit de la démocratie, La République des idées, Paris.

Blondiaux L., Sintomer Y., 2009, « L'impératif délibératif », Rue Descartes 2009(1), n 63, p. 2838.

Bourg D., Whiteside K., 2010, Vers une démocratie écologique. Le citoyen, le savant et le politique, Paris, Seuil.

Bourg D. (ss. dir.), 2011, Pour une 6e République écologique, Odile Jacob.

Bouvier A., 2007, «Démocratie délibérative, démocratie débattante, démocratie participative », Revue européenne des sciences sociales [En ligne], XLV-136, p. 5-34.

Bozonnet J.-P., 2012, «La sensibilité écologique », in Barbier R. et al. (dir.), Manuel de.., p. 147162.

Callon M., Lascoumes P., Barthe Y., 2001, Agir dans un monde incertain. Essi sur la démocratie technique, Seuil.

Cerutti S., 2011, « À rebrousse-poil » : dialogue sur la méthode », Critique 2011(6), n769-770, p. 564-575.

Chaxel S., Fiorelli C., Moity-Maïzi P., 2014, « Les récits de vie : outils pour la compréhension etcatalyseurs pour l'action », ¿ Interrogations ?, n¹7 [en ligne].

DialTer, 2012, Restauration hydraulique et écologique du bassin versant Brevenne-Turdine. Mission de dialogue territorial, Réponse à consultation.

Dziedzicki J.-M., 2015, «Quelles réponses aux conflits d'aménagement? De la participation publique à la concertation », Participations 2015(3), p. 145-170.

Hatzfeld H., ?, «Au nom de quoi ? Légitimités ordinaires et légitimités politiques au cœur de la participation » in L. Blondiaux, J.M. Fourniau, L. Monnayer-Smith, C. Neveu (dir.), La démocratie participative pour quoi faire? Un bilan critique des recherches sur les effets de la participation en démocratie, à paraître.

Fourniau J.-M., 2007, « L'expérience démocratique des "citoyens en tant que riverains" dans les conflits d'aménagement », Revue européenne des sciences sociales [En ligne], XLV-136, p. 149179.

Fourniau J. M. (dir.) et al., 2012, La portée de la concertation. Modélisation sociologique des effets de la participation du public aux processus décisionnels, rapport de recherche pour le programme CDE, IFSTTAR et MEDDE.

Fraser N., 2011, Qu'est-ce que la justice sociale? Reconnaissance et redistribution, La Découverte, Paris.

Girard C., et Le Goff A. (dir.), 2010, La Démocratie délibérative. Anthologie de textes fondamentaux, p. 11-112.

Ingold A., 2011, « Gouverner les eaux courantes en France au XIXe siècle Administration, droits et savoirs », Annales. Histoire, Sciences Sociales 2011(1), p. 69-104.

Knorr Cetina K., 1999, Epistemic Cultures: How the Sciences Make Knowledge, Cambridge, 
Harvard University Press.

Lascoumes P., 1995, «Les arbitrages publics des intérêts légitimes en matière d'environnement », Revue française de science politique, $\mathrm{n}^{\circ} 3$, p. 396-419.

Lascoumes P., Le Bourhis J.P., 1998, « Le bien commun comme construit territorial. Identités d'action et procédures », Politix 11(42), p. 37-66.

Latour B., 1999, Politiques de la nature, comment faire entrer les sciences en démocratie, Paris, La Découverte.

Le Bourhis J.-P., 2003, « Complexité et trajectoires d'apprentissage dans l'action publique. Les instruments de gestion durable des ressources en eau en France et au Royaume-Uni », Revue internationale de politique comparée, vol. 10, p. 161-175.

Le Bourhis J.-P., 2007, « Du savoir cartographique au pouvoir bureaucratique. Les cartes des zones inondables dans la politique des risques (1970-2000) », Genèses 2007(3), n 68, p. 75-96.

Manin B., 1985, «Volonté générale ou délibération. Esquisse d'une théorie de la délibération politique », Le Débat, 33, p. 72-93.

Manin B., 2002, «L'idée de démocratie délibérative dans la science politique contemporaine », Entretien avec Loïc Blondiaux, Politix, 15(57), p. 37-55.

Mermet L., Dubien I, Emerit A, Laurans Y, 2004, « Les porteurs de projets face à leurs opposants : six critères pour évaluer la concertation en aménagement $»$, Politique et Management Public, vol. 22, p.1-22.

Mermet L., 2015, « Que peux t-on encore attendre de la concertation pour l'action environnementale?» in L. Mermet, D. Lesalles (dir.), Environnement : la concertation apprivoisée, contestée, dépassée ? De Boek, Louvain La Neuve, Belgique, p. 17-33.

Mouffe C., 2016, L'illusion du consensus, Albin Michel.

Passeron J-C., 1990, « Biographies, flux, itinéraires, trajectoires », Revue française de sociologie, 31(1), p. 3-22.

Rancière J., 2011, « De la vérité des récits au partage des âmes », Critique 2011(6), n 769-770, p. 474-484.

Revel M., Blatrix C., Blondiaux L., Fourniau J.-M., Hérard-Dubreuil B., Lefebvre R. (dir.), 2007, Le débat public : une expérience française de démocratie participative, Paris, La Découverte.

Rosanvallon P., 2008, La légitimité démocratique. Impartialité, réflexivité, proximité, Seuil.

Simard L., L. Lepage, J.M. Fourniau, M. Gariepy et M. Gauthiere (dir.), 2006, Le débat public en apprentissage. Aménagement et environnement, L'Harmattan.

Syndicat de rivières Brévenne Turdine, 2015, Création d'ouvrages de ralentissement dynamique et travaux de restauration écologique, Dossier de déclaration de projet.

Talpin J., 2010, « Ces moments qui façonnent les hommes. Éléments pour une approche pragmatiste de la compétence civique », Revue française de science politique, vol. 60, p. 91-115.

Touzard H., 2006, "Consultation, concertation, négociation. Une courte note théorique », Négociations 2006(1), n5, p. 67-74.

Urfalino P., 2007, «La décision par consensus apparent. Nature et propriétés », Revue européenne des sciences sociales [En ligne], XLV-136, p. 47-70.

Warin P., Labranche S., 2006, La concertation dans l'environnement ou le besoin de recourir aux sciences sociales, Programme "Concertation, Décision, Environnement", Ministère de l'Environnement et du Développement Durable, p. 80-84. 